\title{
Advantage of Introducing Quantitative Light-Induced Fluorescence in School Dental Checkups
}

\author{
Koji Watanabe ${ }^{1}$, Takashi Sasabe ${ }^{2}$, Akihiro Nakamura ${ }^{2}$, Kosuke Eda², Kosuke Tanase ${ }^{2}$, \\ Hidefumi Ikeda ${ }^{2}$, Naoko Ohata ${ }^{2}$, Yukiko Minohara', Kenshi Maki ${ }^{1}$, Shigeru Watanabe ${ }^{2}$ \\ ${ }^{1}$ Division of Developmental Stomatognathic Function Science, Department of Health Promotion, Kyushu Dental University, \\ Kitakyushu, Japan \\ ${ }^{2}$ Division of Pediatric Dentistry, Department of Human Development and Fostering, Meikai University School of Dentistry, \\ Sakado, Japan \\ Email: *r17watanabe2@fa.kyu-dent.ac.jp
}

How to cite this paper: Watanabe, K., Sasabe, T., Nakamura, A., Eda, K., Tanase, K., Ikeda, H., Ohata, N., Minohara, Y., Maki, K. and Watanabe, S. (2018) Advantage of Introducing Quantitative LightInduced Fluorescence in School Dental Checkups. Health, 10, 1095-1106.

https://doi.org/10.4236/health.2018.108083

Received: July 19, 2018

Accepted: August 25, 2018

Published: August 28, 2018

Copyright $(9) 2018$ by authors and Scientific Research Publishing Inc. This work is licensed under the Creative Commons Attribution International License (CC BY 4.0).

http://creativecommons.org/licenses/by/4.0/

\begin{abstract}
Background: Caries detection in school oral examinations insufficient accuracy. Objective: To evaluate advantages of introducing quantitative lightinduced fluorescence-digital (QLF-D) in school oral examinations. Methods: Experiment No. 1. Early demineralized lesions in the upper and lower incisors and canines were visually inspected by three dentists and by QLF-D. The numbers of tooth planes with early demineralized lesions were compared between the methods. Experiment No. 2. Approximal demineralized lesions in molars were assessed by visual inspection, $\mathrm{x}$-ray imaging, and QLF-D. The numbers of tooth planes with demineralized lesions were compared among the methods. Experiment No. 3. Plaque distribution was evaluated by QLF-D and a traditional staining method. The ratio of the diameter of plaque to tooth crown in the tooth axis direction in each method was calculated. The results were evaluated by Pearson's correlation coefficient analysis and Bland-Altman plot. Results: Experiment No. 1. The three dentists found 0.67 tooth planes on average. QLF-D found 22 tooth planes with early demineralized lesions in the same samples. Experiment No. 2. Fourteen approximal tooth planes of molars were found to have demineralized lesions by $\mathrm{x}$-ray imaging. QLF-D detected 71.4\% of the tooth planes out of the 14, whereas visual inspection found $7.1 \%$. Experiment No. 3. The Pearson's correlation coefficient for the evaluations of plaque distribution between the QLF-D and traditional staining methods was $0.77(\mathrm{P}<0.001)$. No statistically significant systematic error was found through the Bland-Altman Plot analysis. Conclusion: The results support introduction of QLF-D for use in school dental examinations.
\end{abstract}




\section{Keywords}

Oral Checkup, Early Demineralized Lesion, Approximal Demineralized Lesion, Quantitative Light-Induced Fluorescence-Digital

\section{Introduction}

Objective, safe, and easy-to-use means of evaluating demineralized lesions have not been available outside of dental offices, so dental checkups at schools must primarily rely on visual inspection in Japan. However, dentists have to perform visual inspections of numerous students with insufficient time and light sources. Consequently, the accuracy of school dental checkups in Japan depend on the skill of individual dentist. When there is an obvious cavity on the tooth surface, it can be detected easily, but if the demineralized lesion is hidden in the approximal region that cannot be seen directly or is in its early stage, it cannot be found through visual inspection in such poor surroundings [1] [2] [3] [4] [5]. According to a survey by the Japanese government, the caries prevalence ratio decreased to $35.3 \%$ among $\leq 14$-year-old Japanese children in 2016. This finding indicated that many children do not have an obvious cavity in their mouth and that dentists are expected to detect early demineralized lesions, which are more common than cavities but can be found only by professionals. Recently, some laser or fluorescence devices used to evaluate carious lesions have been developed. Although in vitro and in vivo studies have reported the reliability of these devices [6] [7] [8], they have not yet been introduced for use in school dental checkups. In this study, with the goal of introducing quantitative light-induced fluorescence-digital (QLF-D) into school dental checkups, the abilities to detect early carious lesions and evaluate plaque distribution on the tooth surface between traditional methods and QLF-D were compared in available surroundings as school dental checkups. This study protocol was evaluated and approved by the ethics committee of Meikai University (approval number: A1305).

\section{Materials and Methods}

This study was carried out from May 2014 to March 2016.

\subsection{Detection of Early Demineralized Lesions in Incisors and Canines}

Three dentists who had professional backgrounds of $>3$ years performed visual inspections. They inspected early demineralized lesions in incisors and canines in 10 child patients who visited our clinic for regular dental checkup. The child patients were six boys and four girls whose age ranged from 6 to 14 years. In this visual inspection, early demineralized lesions were defined as demineralized lesions that had a shade abnormality, but did not have any cavities. After the visual inspection, images of the teeth were taken by QLF-D under the following con- 
ditions: shutter speed of $1 / 60$ second, aperture value of 5.6 , ISO speed of 1600 [9], and the distance between the lens and the object was $10 \mathrm{~cm}$. The images were taken approximately vertically to the object in a dark room. The child patients were sitting on a chair while the images were taken. The numbers of tooth planes that were diagnosed as having early demineralized lesions by visual inspection and QLF-D were compared.

\subsection{Detection of Approximal Dental Caries in Molars}

Demineralized lesions of approximal planes in upper and lower molars excluding distal planes in posterior end molars (48 surfaces) were visually inspected by three dentists who had professional backgrounds of $>3$ years. Following the visual inspection, the same regions were analyzed by QLF-D under the same conditions as in the detection of early demineralized lesion in incisors and canines. Then, dental radiographs were taken and interpreted. In dental radiographs, an early demineralized lesion was defined as a radiolucent lesion within the enamel (C1) because dental caries is recognized as a radiolucency in a radiograph in daily clinical practice when the lesion becomes $\mathrm{C} 1$ or severer [10].

\subsection{Comparison of Plaque Evaluation between Traditional Staining Method and QLF-D}

Five adult males without past and present systemic illness history whose ages ranged from 27 to 29 years participated in the study as test subjects. The subjects were asked to forego teeth brushing for 60 hours. At 60 -hours, images of the upper and lower central incisors, lateral incisors, and canines were taken by QLF-D under the following conditions: shutter speed of 1/60 second, aperture value of 5.6, ISO speed of 1600 [9], and the distance between the lens and the object was $10 \mathrm{~cm}$. The images were taken approximately vertically to the object in a dark room. The subjects were sitting on a chair while the images were taken. After taking the images by QLF-D, plaque was stained by using a traditional staining method (Dent. Liquid Plaque Tester: Lion Corporation, Tokyo, Japan), and oral photographs were taken by using a single-lens reflex camera. Plaque distribution was evaluated in the images taken by QLF-D and the camera, respectively. The ratio of the height in the tooth-axis direction of the plaquedetected area to the crown dimension was calculated for the respective tooth in both kinds of images based on the Oral Health Index [11] (Figure 1), and the ratio was compared by Bland-Altman plot [12] [13] and Pearson's correlation coefficient analysis to determine if there was systematic error between the plaque evaluations by the staining method and QLF-D.

\section{Results}

\subsection{Detection of Early Demineralized Lesions in Incisors and Canines}

Table 1 shows a comparison of the mean numbers of early carious lesions 
Table 1. Results of examinations by visual inspection and QLF-D.

\begin{tabular}{ccc}
\hline & & (Unit: Tooth Planes) \\
\hline Patients & Visual Inspection & QLF-D \\
\hline A & 0 & 3 \\
B & 0 & 0 \\
C & 0 & 8 \\
D & 0 & 3 \\
E & 0.67 & 4 \\
F & 0 & 2 \\
G & 0 & 0 \\
H & 0 & 1 \\
I & 0 & 1 \\
J & 0 & 0 \\
\hline
\end{tabular}

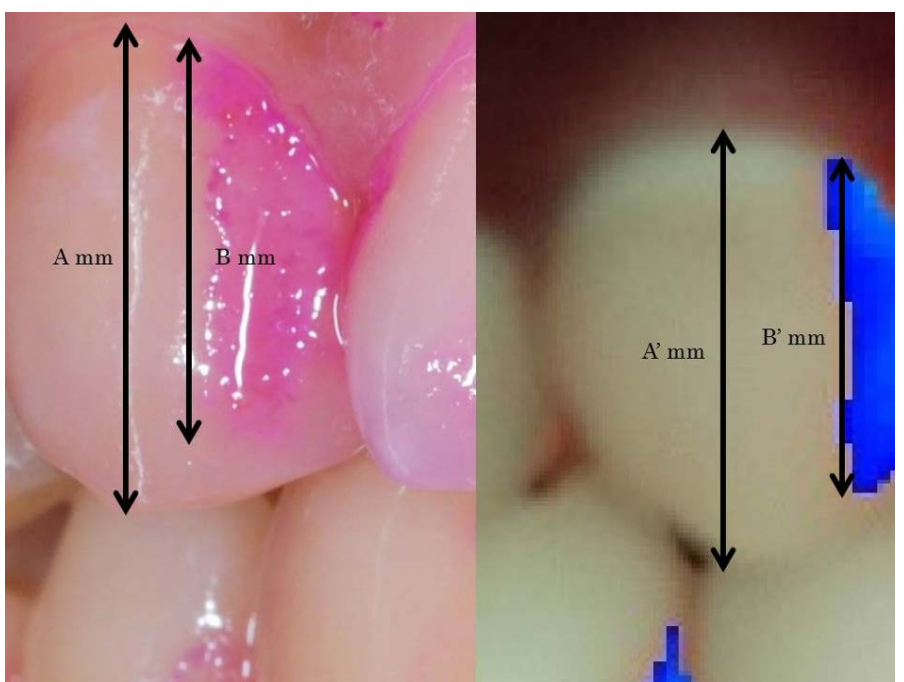

Figure 1. Comparison of evaluations of plaque distribution between QLF-D and the usual staining method. The ratio of the diameter of the area that was determined to be covered with plaque to the tooth crown in the tooth axis direction (shown as $\mathrm{B}(\mathrm{mm}) / \mathrm{A}(\mathrm{mm})$ and $B^{\prime}(\mathrm{mm}) / \mathrm{A}^{\prime}(\mathrm{mm})$ in Figure 3) in each method was calculated. The result was evaluated by Bland-Altman plot analysis.

detected through visual inspection or QLF-D. Two of the three dentists who had professional backgrounds of $>3$ years found a tooth plane with an early demineralized lesion in only one participating child through visual inspection. On the other hand, QLF-D found early demineralized lesions in seven participating children out of 10. QLF-D detected four tooth planes with early demineralized lesions in a child who had been determined to have had an average number of 0.67 tooth planes with early demineralized lesions through the visual inspection.

\subsection{Detection of Approximal Dental Caries in Molars}

Table 2 shows the numbers of tooth surfaces with molar demineralized lesions in molars found through visual inspection, QLF-D, and radiographs. 
Table 2. Results of examination by visual inspection, QLF-D, and X-ray.

\begin{tabular}{cc}
\hline Results of Examination & Tooth Planes \\
\hline Visual Inspection (-), QLF-D (-), X-ray (-) & 13 \\
Visual Inspection (+), QLF-D (+), X-ray (+) & 0 \\
Visual Inspection (+), QLF-D (-), X-ray (-) & 10 \\
Visual Inspection (-), QLF-D (+), X-ray (-) & 10 \\
Visual Inspection (-), QLF-D (-), X-ray (+) & 4 \\
Visual Inspection (-), QLF-D (+), X-ray (+) & 9 \\
Visual Inspection (+), QLF-D (-), X-ray (+) & 1 \\
Visual Inspection (+), QLF-D (+), X-ray (-) & 1 \\
\hline
\end{tabular}

QLF-D detected nine out of 14 tooth planes that had demineralized lesions in which the lesion was found by radiography. On the other hand, no tooth surface with a demineralized lesion was found through visual inspection out of the 14 tooth planes. Of the tooth planes in which lesions were found through visual inspection, 7.1\% were diagnosed as being sound by QLF-D. In the same way, 7.1\% of the tooth planes in which demineralized lesions were found in radiography were found to have demineralized lesions through visual inspection, which meant that $92.9 \%$ of the approximal tooth planes with demineralized lesions were missed in the visual inspection.

Demineralized lesions were found in 25 tooth planes through radiography or QLF-D. QLF-D detected demineralized lesions in 10 of the 25 tooth planes, whereas radiography found four tooth planes with demineralized lesions. Both QLF-D and radiography detected the lesions in nine out of the 25 tooth planes.

\subsection{Comparison of Plaque Adhesion}

Figure 2(a) (method comparison graph) and Figure 2(b) (Bland-Altman plot) show comparisons of plaque adhesion between the traditional staining method and QLF-D. The Pearson's correlation coefficient between the two methods was $0.77(\mathrm{P}<0.001)$ (Figure 2(a)). In Figure 2(b), the 95\% confidence interval $(95 \%$ $\mathrm{CI}$ ) was $-0.023 \pm 0.082$. The $\mathrm{t}$-value between values of the abscissa and ordinate axes in the Bland-Altman plot was -2.31 .

\section{Discussion}

\subsection{QLF-D}

When a tooth is illuminated with blue light (wavelength of $405 \mathrm{~nm}$ ), the tooth emits fluorescence especially at the enamel-dentin junction. This fluorescence loses energy, increases in wavelength, and travels through enamel. QLF-D consists of a single-lens reflex camera equipped with a blue and white light-emitting diode (LED) and personal computer in which software for analysis is installed (Figure 3). The single-lens reflex camera takes pictures of the tooth under light emitted from a blue LED. From the photograph, the computer software was used 


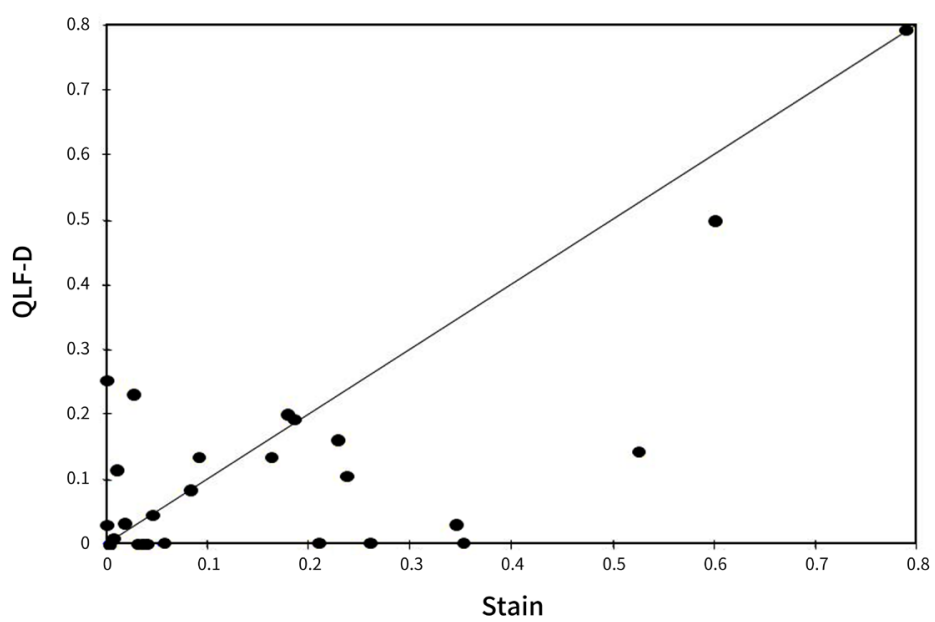

(a)

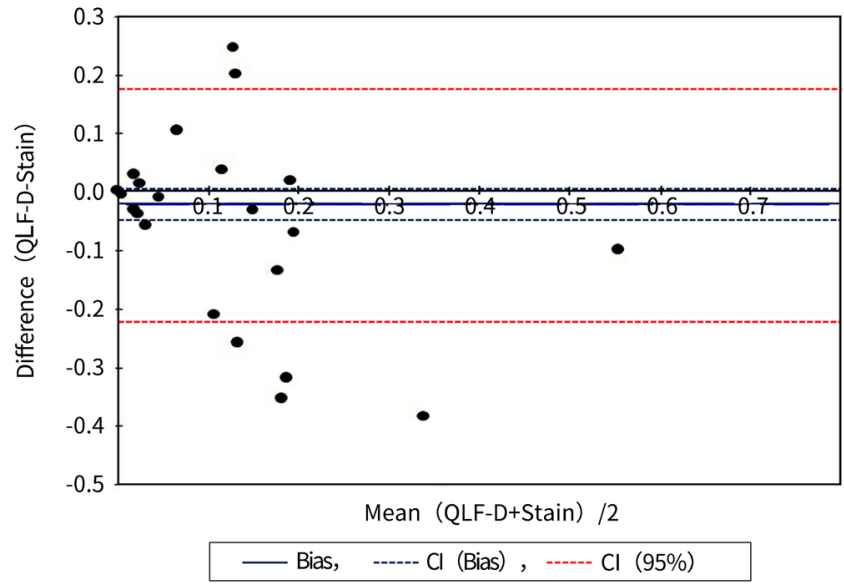

(b)

Figure 2. (a) Scatter diagram of the results by QLF-D and the usual staining method. Ratios of the height of the stained area (B or B' in Figure 3) to tooth crown (A or A' in Figure 3 ). The values obtained by the usual staining method were assigned to the $\mathrm{x}$-axis, and those by the QLF-D were assigned to the y-axis. The linear equation was $y=0.9348 x+$ 0.0264. The Pearson's coefficient of correlation was 0.77 ( $p<0.001)$. (b) Evaluation by Bland-Altman plot. The mean value of results obtained by the two kinds of plaque detection methods was assigned to the $\mathrm{x}$-axis, and the differences in the results were assigned to the $y$-axis.

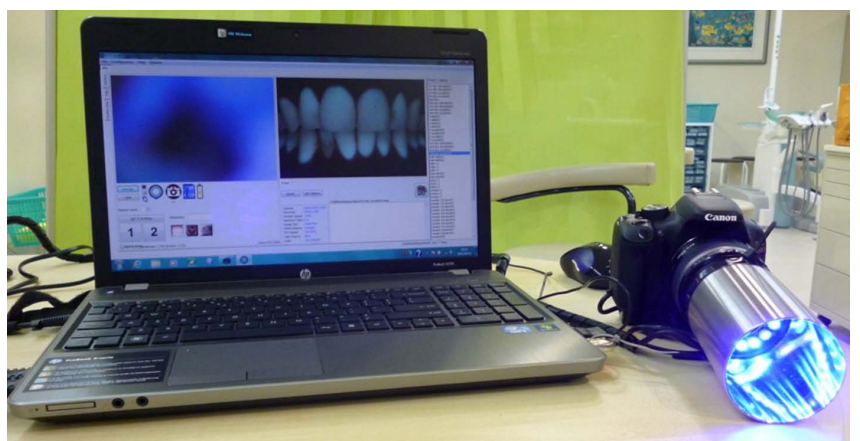

Figure 3. QLF-D. QLF-D consists of a single-lens reflex camera that has blue and white LED lights and a laptop to analyze the obtained data. 
to measure the volume of fluorescence emitted by the enamel in response to the blue light from the LED. The volume of fluorescence emitted from sound enamel is defined as F1. The fluorescent light that travels through a demineralized lesion is reduced by diffuse reflection, which is defined as F2 (Figure 4). The software calculates the difference between F1 and F2 $(\Delta \mathrm{F}=\mathrm{F} 2-\mathrm{F} 1)$ and the dimension where the reduced fluorescence was observed (S), and then determines mineral loss by multiplying $\Delta \mathrm{F}$ by $\mathrm{S}(\Delta \mathrm{Q})$ [14] [15]. Since $\Delta \mathrm{F}$ is a negative value, $\Delta \mathrm{Q}$ is also negative. In this study, $\Delta \mathrm{F}$ was used to determine the presence or absence of a demineralized lesion because three-dimensional diagnosis was not possible either by visual inspection or radiography.

\subsection{Examination of Early Demineralized Lesions in Incisors and Canines}

As shown in Table 1, one or more tooth planes with early demineralized lesions were found by QLF-D in seven child patients. On the other hand, two of the three dentists who had professional backgrounds of $>3$ years found only one tooth plane with an early demineralized lesion in one child patient. Even in the child patients, visual inspection found only 0.67 tooth planes with early demineralized lesions on average, whereas QLF-D detected four tooth planes with early demineralized lesion. These results suggested that visual inspection missed early demineralized lesions in incisors and canines even if it was performed by dentists with professional backgrounds of $>3$ years. Moreover, visual inspection was performed by using a dental chair and the type of light commonly used in daily clinical practice, so the results suggested that early demineralized lesions can be

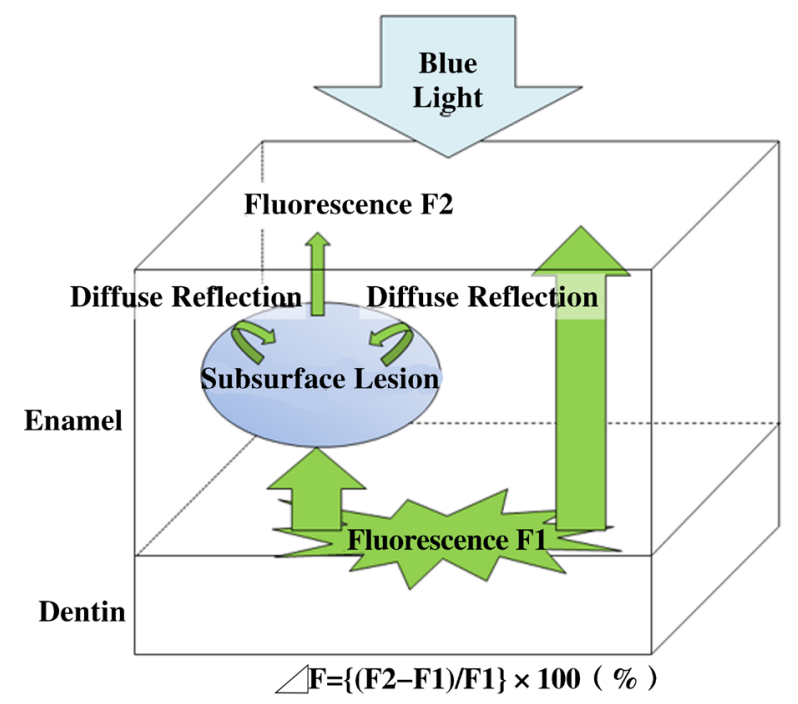

Figure 4. Mechanism of QLF-D. The enamel-dentin junction emits fluorescence when the tooth is illuminated with blue light. When enamel is sound, the amount of fluorescence is determined as F1. If there is a demineralized lesion in the enamel, the amount of reflected fluorescence, $\mathrm{F} 2$, is less than $\mathrm{F} 1$ because of diffuse reflection. $\Delta \mathrm{F}$, which is determined as the difference between F1 and F2, was adopted as a parameter of enamel mineral amount in this study. 
missed in regular school checkups, which are performed at schools with insufficient light sources and without dental chairs. Gimenez et al. [16] searched previous studies and reported that visual inspection appeared to be effective in caries detection. Additionally, in Scotland, dentists who perform dental checkups for children participate in the National Dental Inspection Programme, and they diagnose caries with only a small error rate attributed to accident [17]. However, the lesions in a report by Gimenez et al. were caries lesions beyond the early stage. The training content of the National Dental Inspection Programme was also intended for carious legions with a cavity and is based on diagnosis of a decayed, missing, and filled tooth. So, the subjects of this study were in a far earlier stage of demineralization than that in the previous studies.

\subsection{Inspection of Approximal Carious Lesions}

Radiography is widely used in determining the prognosis of dental caries. Thus, it was required that QLF-D had at least the same caries sensing capability as that of radiography in this study. So, the accuracies of visual inspection and QLF-D were evaluated in tooth planes in which demineralized lesions were found by radiography. Visual inspection detected the demineralized lesions in $7.1 \%$ out of the tooth planes, whereas QLF-D detected the lesions in $64.3 \%$ of them. The results suggested that the combined application of QLF-D and visual inspection was more effective in approximal caries detection in molars. Ko HY et al. [9] reported in their in vitro study that QLF-D had detectability of approximal caries coequal to that of bite-wing projection or visual inspection. However, in this in vivo study, detectability of visual inspection was less than that of radiography or QLF-D, which suggested that detection of approximal caries is more difficult in real oral cavities than in an artificial environment. In this study, QLF-D detected demineralized lesions in $64.3 \%$ of the approximal tooth planes out of those in which the lesions were found through radiography. In other words, QLF-D did not detect demineralized lesions in $35.7 \%$ of the approximal tooth planes out of those in which the lesions were found through radiography. A technical cause could affect the false-negative result in approximal caries detection by QLF-D. The quantity of fluorescence was determined on the basis of images taken by a single-lens reflex camera in the QLF-D system. The image was usually focused on the center of the frame. Image sharpness was the highest at the center of the image and decreased with increasing distance from the center. There was a possibility that detection would because of reduced fluorescence due to the decrease in image sharpness if the dimensions of the lesions were small. Such a technical cause could be eliminated by taking images focused on different points. In the present study, QLF-D detected approximately nine times as many approximal lesions as that detected by visual inspection; hence the results suggested that QLF-D was an effective means of caries detection in oral checkups.

Since it was reported that there was a high correlation between QLF-D and pathological examination of enamel in caries detection [18] [19], the numbers of tooth planes that were diagnosed as having carious lesions by QLF-D or/and ra- 
diography were counted. Carious lesions were found only by QLF-D in $40.0 \%$ of the candidate tooth planes. Carious lesions were found only by radiography in $16.0 \%$ out of the candidate tooth planes. These findings indicated that there were approximal carious lesions in the early stage that were not detected by radiography, but were found by QLF-D.

In Japan, dentists visit schools and perform oral checkups regularly; they have to make a diagnosis in approximately 30 seconds per student with a poor light source, otherwise they cannot accomplish their assignment within the schedule. If dentists introduce QLF-D for use in oral checkups at schools, they can bring the images home, analyze them objectively without a tight time limit, and give notice later as done for hematological examination. QLF-D detects carious lesions in the very early stage, at which point they should be controlled instead of being drilled and filled. Jallad et al. (2015) [18] reported that carious lesions on occlusal surfaces could be found by visual inspection if the $\Delta \mathrm{F}$ value was $<-7 \%$, which suggested that if the $\Delta \mathrm{F}$ value of the lesion is $>-7 \%$, the lesion cannot be detected through traditional visual inspection in a school oral checkup, which resulted in missing an opportunity for receiving habit guidance and remineralization therapy from a dentist. Detection of such lesions with $\Delta \mathrm{F}$ values $>-7 \%$ will be one of the merits of introducing QLF-D into oral checkups at schools from the viewpoint of controlling demineralized lesions at the very early stage.

\subsection{Comparison of Plaque Adhesion}

Although QLF-D was originally developed for evaluation of demineralized lesions in the early stages, it is also known that porphyrin in plaque also reflects fluorescence when it is illuminated with blue light by QLF-D [20] [21]. In the present study, the ratio of the height in the tooth-axis direction of the plaque detected area to the crown dimension was adopted as the parameter of plaque adhesion. The parameter was based on the Oral Health Index [11], which is used to assess plaque distribution in daily clinical practice. Moreover, it was considered that accidental error due to shooting angle between the intraoral photograph and image of QLF-D was corrected by calculating the "ratio" of the height in the tooth-axis direction of the plaque-detected area to the crown dimension.

In Figure 2(a), the Pearson's correlation coefficient between the two methods was 0.77 which included moderately strong correlation. However, "strong correlation" does not necessarily mean "statistically the same." There could still be "systemic error" between the two methods. In this study, a Bland-Altman plot was generated to make sure that there was no systemic error between the two methods. In Figure 2(b), the abscissa of the Bland-Altman graph is the mean measured value of the staining method and QLF-D in each tooth, and the ordinate of the Bland-Altman graph is the difference in the measured values between the staining method and QLF-D for each tooth. In the graph, the 95\% CI included zero; hence, no fixed bias was found between the staining method and QLF-D. Moreover, the t-value for the differences between values of the abscissa and ordinate axes in the Bland-Altman graph was -2.31 . The absolute value of 
the t-value was $>2$; hence, the result indicated that there was no proportional bias between staining method and QLF-D. These findings indicated that there was no systemic bias in plaque detection between the staining method and QLF-D.

The results of the Pearson's correlation coefficient analysis and Bland-Altman plot suggested that there was no statistical difference in plaque detection between the staining method and QLF-D.

\subsection{Advantages and Disadvantages of Introducing QLF-D into School Oral Checkups}

In Japan, dentists have to see approximately two students per minute with poor light sources to complete their duties in time when they provide oral checkups at schools. The oral checkup is usually performed only by visual inspection. As a result, it has been said that the accuracy of school oral checkups is insufficient. In this study, demineralized lesions and plaque adhesion were evaluated by QLF-D in available surroundings in school dental checkups, and the findings indicated that QLF-D was effective in such surroundings. If QLF-D is introduced for use in school oral checkups, the dentists can obtain a sufficient light source by using the flash of the single-lens reflex camera and will only have to take photographs at school. They can then take the images back to their offices and make a diagnosis objectively without a time constraint. QLF-D can detect demineralized lesions at the very early stage, so dentists can provide proper advice to school children through oral checkups before the lesions deteriorate further.

One slight disadvantage of QLF-D is that dentists have to have sufficient skill to take proper images, but the photographic process is mostly automated.

\section{Conclusions}

1) Detection of demineralized lesions at the very early stage through visual inspection was very difficult.

2) Detection of demineralized lesions in the approximal plane through visual inspection was also difficult.

3) QLF-D was a very effective means of detecting both an early demineralized lesion and a demineralized lesion in the approximal tooth plane.

4) QLF-D detected an early demineralized lesion that was not found by visual inspection or radiography. Detection by QLF-D of an early demineralized lesion could be useful for encouraging children to receive daily habits and oral hygiene instruction.

5) QLF-D showed good accuracy for plaque detection that was statistically equal to that of a traditional staining method.

6) QLF-D was found to be an effective means of detecting plaque and demineralized lesions in school oral checkups.

\section{Conflicts of Interest}

The authors declare no conflicts of interest regarding the publication of this paper. 


\section{References}

[1] Lussi, A. (1993) Comparison of Different Methods for the Diagnosis of Fissure Caries without Cavitation. Caries Research, 27, 409-416.

https://doi.org/10.1159/000261572

[2] Rocha, R.O., Ardenghi, T.M., Oliveira, L.B., Rodrigues, C.R. and Ciamponi, A.L. (2003) In Vivo Effectiveness of Laser Fluorescence Compared to Visual Inspection and Radiography for the Detection of Occlusal Caries in Primary Teeth. Caries Research, 37, 437-441. https://doi.org/10.1159/000073396

[3] Shimada, Y., Sadr, A., Burrow, M.F., Tagami, J., Ozawa, N. and Sumi, Y. (2010) Validation of Swept-Source Optical Coherence Tomography (SS-OCT) for the Diagnosis of Occlusal Caries. Journal of Dentistry, 38, 655-665. https://doi.org/10.1016/j.jdent.2010.05.004

[4] Chawla, N., Messer, L.B., Adams, G.G. and Manton, D.J. (2012) An In Vitro Comparison of Detection Methods for Approximal Carious Lesions in Primary Molars. Caries Research, 46, 161-169. https://doi.org/10.1159/000337099

[5] Hoskin, E.R. and Keenan, A.V. (2016) Can we trust visual methods alone for detecting caries in teeth? Evidence Based Dentistry, 17, 41-42. https://doi.org/10.1038/sj.ebd.6401165

[6] Silvertown, J.D., Wong, B.P., Abrams, S.H., Sivagurunathan, K.S., Mathews, S.M. and Amaechi, B.T. (2016) Comparison of the Canary System and DIAGNOdent for the In Vitro Detection of Caries under Opaque Dental Sealants. Journal of Investigative and Clinical Dentistry, 8.

[7] Bizhang, M., Wollenweber, N., Singh-Hüsgen, P., Danesh, G. and Zimmer, S. (2016) Pen-Type Laser Fluorescence Device versus Bitewing Radiographs for Caries Detection on Approximal Surfaces. Head \& Face Medicine, 12, 30. https://doi.org/10.1186/s13005-016-0126-9

[8] Melo, M., Pascual, A., Camps, I., Del Campo, Á. and Ata-Ali, J. (2017) Caries Diagnosis Using Light Fluorescence Devices in Comparison with Traditional Visual And Tactile Evaluation: A Prospective Study in 152 Patients. Odontology, 105, 283-290.

[9] Ko, H.Y., Kang, S.M., Kim, H.E., Kwon, H.K. and Kim, B.I. (2015) Validation of Quantitative Light-Induced Fluorescence-Digital (QLF-D) for the Detection of Approximal Caries in Vitro. Journal of Dentistry, 43, 568-575. https://doi.org/10.1016/j.jdent.2015.02.010

[10] Evans, R.W. and Dennison, P.J. (2009) The Caries Management System: An Evidence-Based Preventive Strategy for Dental Practitioners. Application for Children and Adolescents. Australian Dental Journal, 54, 381-389. https://doi.org/10.1111/j.1834-7819.2009.01165.x

[11] Burke, F.J.T., Busby, M., McHugh, S., Delargy, S., Mullins, A. and Matthews, R. (2003) Evaluation of an Oral Health Scoring System by Dentists in General Dental Practice. British Dental Journal, 194, 215-218. https://doi.org/10.1038/sj.bdj.4809915

[12] Giavarina, D. (2015) Understanding Bland Altman Analysis. Biochemia Medica (Zagreb), 25, 141-151. https://doi.org/10.11613/BM.2015.015

[13] Bland, J.M. and Altman, D.G. (1986) Statistical Methods for Assessing Agreement between Two Methods of Clinical Measurement. Lancet, 1, 307-310.

[14] Han, S., Fan, Y., Zhou, Z., Tu, H., Li, D., Lv, X., Ding, L. and Zhang, L. (2016) Promotion of Enamel Caries Remineralization by an Amelogenin-Derived Peptide in a 
Rat Model. Archives of Oral Biology, 73, 66-71.

https://doi.org/10.1016/j.archoralbio.2016.09.009

[15] Savas, S., Kavrik, F. and Kucukyilmaz, E. (2016) Evaluation of the Remineralization Capacity of CPP-ACP Containing Fluoride Varnish by Different Quantitative Methods. Journal of Applied Oral Science, 24, 198-203.

https://doi.org/10.1590/1678-775720150583

[16] Gimenez, T., Piovesan, C., Braga, M.M., Raggio, D.P., Deery, C., Ricketts, D.N., Ekstrand, K.R. and Mendes, F.M. (2015) Visual Inspection for Caries Detection: A Systematic Review and Meta-Analysis. Journal of Dental Research, 94, 895-904. https://doi.org/10.1177/0022034515586763

[17] O’Keefe, E.J., McMahon, A.D., Jones, C.M., Curnow, M.M. and Macpherson, L.M. (2016) Evaluation of Dental Therapists Undertaking Dental Examinations in a School Setting in Scotland. Community Dentistry and Oral Epidemiology, 44, 515-522. https://doi.org/10.1111/cdoe.12244

[18] Jallad, M., Zero, D., Eckert, G. and Ferreira Zandona, A. (2015) In Vitro Detection of Occlusal Caries on Permanent Teeth by a Visual, Light-Induced Fluorescence and Photothermal Radiometry and Modulated Luminescence Methods. Caries Research, 49, 523-530. https://doi.org/10.1159/000437214

[19] Lenzi, T.L., Piovesan, C., Mendes, F.M., Braga, M.M. and Raggio, D.P. (2016) In Vitro Performance of QLF System and Conventional Methods for Detection of Occlusal Caries around Tooth-Colored Restorations in Primary Molars. International Journal of Paediatric Dentistry, 26, 26-34. https://doi.org/10.1111/ipd.12154

[20] Kim, Y.S., Lee, E.S., Kwon, H.K. and Kim, B.I. (2014) Monitoring the Maturation Process of a Dental Microcosm Biofilm Using the Quantitative Light-Induced Fluorescence-Digital (QLF-D). Journal of Dentistry, 42, 691-696. https://doi.org/10.1016/j.jdent.2014.03.006

[21] Lee, E.S., Kang, S.M., Ko, H.Y., Kwon, H.K. and Kim, B.I. (2013) Association between the Cariogenicity of a Dental Microcosm Biofilm and Its Red Fluorescence Detected by Quantitative Light-Induced Fluorescence-Digital (QLF-D). Journal of Dentistry, 41, 1264-1270. https://doi.org/10.1016/j.jdent.2013.08.021 